\title{
Thyroid Hormone Changes in Euthyroid Patients with Diabetes
}

This article was published in the following Dove Press journal:

Diabetes, Metabolic Syndrome and Obesity: Targets and Therapy

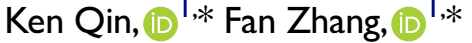 \\ Qinqin Wu,' Zhenrong Liu, (D) \\ Yan Huang,' Jing Tan, ${ }^{2}$ Yiling \\ Zhou, ${ }^{3}$ Zhenmei An, ${ }^{3}$ \\ Shuangqing $\mathrm{Li}^{4}$ Sheyu Li $\mathbb{D}^{3,5,6}$
}

'Physical Examination Center, West China Hospital, Sichuan University, Chengdu 610041, People's Republic of China; ${ }^{2}$ Chinese Evidence-Based Medicine Center CREAT Group, West China Hospital, Sichuan University, Chengdu 61004I, People's Republic of China; ${ }^{3}$ Department of Endocrinology and Metabolism, West China Hospital, Sichuan University, Chengdu 61004I, People's Republic of China; ${ }^{4}$ General Medical Center, West China Hospital, Sichuan University, Chengdu 61004I, People's Republic of China; ${ }^{5}$ Chinese Evidence-Based Medicine Center, Cochrane China Center and MAGIC China Center, West China Hospital, Sichuan University, Chengdu 61004I, People's Republic of China; ${ }^{6}$ Engineering Research Center for Medical Information Technology, Ministry of Education, West China Hospital of Sichuan University, Chengdu 61004I, People's Republic of China

*These authors contributed equally to this work

Correspondence: Sheyu Li Department of Endocrinology and Metabolism, West China Hospital, Sichuan University, Chengdu 61004I, People's Republic of China

Tel +86 13194874843

Email lisheyu@gmail.com

Shuangqing $\mathrm{Li}$

General Medical Center, West China Hospital, Sichuan University, Chengdu 6I004I, People's Republic of China Tel +8618980601354

Email I25959447I@qq.com
Background: Thyroid dysfunction is associated with diabetes, but it is unclear if the thyroid hormone levels change in euthyroid adults with diabetes.

Objective: To investigate the association between thyroid hormone levels and diabetes in euthyroid adults.

Methods: Among the euthyroid adults who underwent health examination in West China Hospital of Sichuan University in 2016, patients with diabetes were identified according to the medical history, fasting blood glucose and HbA1c. Age and sex matched controls were identified from the population. The patients with diabetes group was further divided into two subgroups: patients with newly diagnosed diabetes (NDD) and with previously diagnosed diabetes (PDD). Independent $t$-test and multivariate logistic regression models were used to investigate the difference in the levels of thyroid stimulating hormone (TSH), free thyroxine (FT4), free triiodothyronine (FT3) and the ratio of FT4/FT3 between groups.

Results: We included 32,557 participants, 2,271 with diabetes. Compared to the adults without diabetes, the odds ratios (ORs) per one unit elevation of TSH, FT4, FT4/FT3 ratio and FT3 in patients with diabetes were 0.88 [95\% confidence interval (CI): 0.82-0.95], 1.11 (95\% CI: 1.08-1.14), 2.05 (95\% CI: 1.81-2.32) and 0.85 (95\% CI: 0.78-0.93), respectively. Compared to the NDD group, the ORs per one unit elevation of TSH, FT4, FT4/FT3 ratio and FT3 of the PDD group were 0.81 (95\% CI: 0.71-0.92), 1.08 (95\% CI: 1.04-1.12), 1.76 (95\% CI: 1.49-2.08) and 1.01 (95\% CI: 0.92-1.12), respectively.

Conclusion: In euthyroid adults, diabetes was associated with increased FT4/FT3 ratio, which is linked to the peripheral turnover of the thyroid hormones.

Keywords: diabetes mellitus, thyroid hormones, triiodothyronine, thyroxine

\section{Introduction}

Thyroid hormones play key roles in energy metabolism, body shape, aging, and glucose and lipid metabolism in humans. ${ }^{1-5}$ Accumulated evidence suggested that both underproduction and overproduction of thyroid hormones contributed to the development and the progression of diabetes. ${ }^{6-10}$ Ashwini et al reported that mild hypothyroidism improved glucose tolerance and redox in adults. ${ }^{11}$ Some in vitro and in vivo studies suggested that excessive thyroid hormone might impair the function of islet beta cells, while an appropriate concentration of thyroid hormone could inhibit the apoptosis of islet beta cells and promote the proliferation of islet beta cells. ${ }^{12-14}$ Chaker et al pointed out that low and low-normal thyroid function were risk factors for incident diabetes, especially in individuals with prediabetes. ${ }^{15}$ However, the changes in thyroid hormone levels in euthyroid adults with and without diabetes are still unclear. It is also unknown whether the 
thyroid hormones change in euthyroid patients with diagnosed diabetes as the disease progresses. This study aimed to investigate the association between the levels of thyroid hormones and diabetes among the euthyroid population.

\section{Methods}

\section{Study Design, Settings and Participants}

Our study is a retrospective cross-sectional study, collecting health physical examination data from 1 January 2016 to 31 December 2016 in the Physical Examination Center, West China Hospital of Sichuan University.

We included the participants if they: 1) were aged between 20 and 79 years old; 2) had available data of TSH, FT4, FT3, thyroid autoantibodies [thyroid peroxidase antibody (TPOAb) and thyroglobulin antibody (TGAb)], hemoglobin A1c (HbA1c) and fasting blood glucose (FBG); and 3) agreed to the scientific use of their health data and signed the informed consent.

We excluded the patients if their: 1) TPOAb or TGAb were positive; 2) TSH levels were higher than $4.2 \mathrm{mU} / \mathrm{L}$ or lower than $0.27 \mathrm{mU} / \mathrm{L}$, out of the normal ranges of reference of the laboratory assay; 3) FT4 or FT3 were out of the normal ranges of reference $(12.0 \sim 22.0 \mathrm{pmol} / \mathrm{L}$ and 3.6 7.5 pmol/L, respectively); 4) recorded history included thyroid surgery, diagnosed hypothyroidism, hyperthyroidism or thyroid malignancy; 5) comorbidities included malignancy, coronary artery disease, stroke, chronic obstructive pulmonary disease (COPD) or cirrhosis; and 6) estimated glomerular filtration rate (eGFR) was less than $60 \mathrm{~mL} /\left(\min \cdot 1.73 \mathrm{~m}^{2}\right)$.

\section{Grouping}

Diabetes was identified if the patient had previous history of diagnosed diabetes, $\mathrm{FBG}>7.0 \mathrm{mmol} / \mathrm{L}$ or $\mathrm{HbAlc}$ $>6.5 \% .{ }^{16}$ The previous history of diagnosed diabetes was identified by patient self-report with validated records provided by well-trained physicians. Controls without diabetes were identified by 1:1 matching using sex, age (threshold $\leq 1 \mathrm{yr}$ ) and BMI (threshold $\leq 0.5 \mathrm{~kg} / \mathrm{m}^{2}$ ). The patients with diabetes were further divided into two subgroups: patients with newly diagnosed diabetes (NDD) group if they had elevated FBG $(>7.0 \mathrm{mmol} / \mathrm{L})$ or HbAlc $(>6.5 \%)$ but were free of self-reported previously diagnosed diabetes, and patients with previously diagnosed diabetes (PDD) group if they had history of diagnosed diabetes before the visit.

\section{Data Source}

The data were from the Health Physical Examination Center dataset in West China Hospital of Sichuan University. The comorbidities were routinely collected by physician interviewing. Weight $(\mathrm{kg})$, height $(\mathrm{cm})$, waist circumference (WC, $\mathrm{cm}$ ) and blood pressure were measured. Body mass index (BMI) was calculated using weight $(\mathrm{kg})$ divided by the square of height $\left(\mathrm{m}^{2}\right)$. Thyroid hormones and thyroid autoantibodies were tested using electrochemiluminescence immunoassay (Roche). Other parameters were tested using cobas8000 (Roche) in the clinical laboratory of the hospital, including $\mathrm{HbA1c}$, triglyceride (TG), high-density lipoprotein cholesterol (HDL-C), low-density lipoprotein cholesterol (LDL-C), alanine aminotransferase (ALT), aspartate aminotransferase (AST) and glutamyl transpeptidase (GGT). The eGFR was calculated using the CKD-EPI Creatinine Equation (2009). ${ }^{17}$

\section{Variables}

The first binomial outcome of our study was participants with diabetes vs without diabetes, and the second was patients with NDD vs with PDD. The interested factors included levels of TSH, FT4, FT3 and the ratio of FT4/ FT3. The other collected variables included age, sex, BMI, diastolic blood pressure (DBP), systolic blood pressure (SBP), WC, HbA1c, FBG, TG, HDL-C, LDL-C, ALT, AST, GGT, eGFR, current smoking, current drinking and whether having hypertension.

\section{Statistical Analyses}

The continuous variables, TG, HbA1c, FBG, FT3, HDL-C, ALT, AST, GGT and eGFR, had been tested to be of non-normal distribution by the KolmogorovSmirnov test between groups, patients with vs without diabetes and PDD vs. NDD groups. After these variables were transformed more closely to a normal distribution by $\log$ transformation, an independent $t$-test was used to investigate the difference of these continuous variables between these groups. The comparison of categorized variables was conducted using the chi-squared test. To compare the levels of TSH, FT4, FT3 and the ratio of FT4/FT3 between the patients with diabetes group and the patients without diabetes group, we used three logistic regression models which were: univariate model (Model 1); multivariate model adjusted for DBP and TG (Model 2); and multivariate model adjusted for Model 2+ALT and 
eGFR (Model 3). To compare the four thyroid hormone outcomes between the NDD group and the PDD group, four logistic regression models were performed, including: univariate model (Model 1); multivariate model adjusted for age, sex and BMI (Model 2); multivariate model adjusted for Model 2+DBP and TG (Model 3); and multivariate model adjusted for Model 3+ALT and eGFR (Model 4). The adjusted variables of these models were selected if they are available in our data and typically associated with diabetes or thyroid function. Three multivariate models were performed in our study to explore the robustness of the results. The significance level of all analyses above was considered as 0.05 , and all analyses were performed using SPSS 24.0.

\section{Results}

\section{Baseline Characteristics of the Study Subjects}

We identified 32,557 participants from 170,896 people who underwent physical examinations in 2016 in West China Hospital of Sichuan University. There were 2,271 (7.0\%) cases of diabetes, of which 1,187 cases were NDD and 1,084 cases were PDD. The process of study population selection is shown as a flow chart (Figure 1).

As shown in Table 1, the average age, BMI and the proportion of male of patients with diabetes were higher. After matching by sex, age and BMI, patients with diabetes presented higher FT4, TG, FPG, HbA1c, ALT, GGT, eGFR, SBP, DBP, WC and FT4/FT3 ratio, and more hypertension, drinking and smoking, but lower TSH, FT3, LDL-C and HDL-C compared to those without diabetes $(P<0.01)$. Compared to the NDD group, patients in the PDD group showed higher FT4, FPG, HDL-C and FT4/FT3 ratio $(P \leq 0.01)$ and a higher portion of females $(P=0.03)$, but lower FT3, TG, LDL-C, ALT, AST, GGT, SBP, DBP, BMI and WC, and less alcohol drinking and smoking $(P<0.05)$. There were no statistically significant differences in the levels of TSH, eGFR and HbAlc between NDD and PDD groups $(P>0.05)$.

\section{The Changes of Thyroid Parameters in Patients with Without Diabetes}

As shown in Table 2, diabetes was associated with lower TSH and FT3 but higher FT4 and FT4/FT3 ratio in all three models. Compared to patients without diabetes, the ORs per one unit elevation and their 95\% CIs of TSH, FT4, FT3 and FT4/FT3 in patients with diabetes were 0.88
(0.82-0.95), $1.11(1.08-1.14), 0.85(0.78-0.93)$ and 2.05 $(1.81-2.32)$ in Model 3, respectively $(P<0.001)$.

\section{The Changes of Thyroid Parameters in Newly and Previously Diagnosed Diabetes}

As shown in Table 3, patients in the PDD group presented lower FT3 but higher FT4 and FT4/FT3 ratio in all four models. Compared to the NDD group, the ORs per one unit elevation and their 95\% CIs of FT4, FT3 and FT4/ FT3 in the PDD group were $1.08(1.04-1.12 ; P<0.001)$, $0.81(0.71-0.92 ; P=0.002)$ and $1.76(1.49-2.08 ; P<0.001)$ in Model 4, respectively.

\section{Discussion}

Our cross-sectional population-based study suggested that thyroid hormone profiles were associated with diabetes and its duration in euthyroid adults. The FT4/FT3 ratio elevates significantly in patients with diabetes, especially in those with PDD, indicating diabetes with longer duration may be associated with a lower peripheral turnover of thyroxine $^{18,19}$ and increased chances of developing euthyroid sickness syndrome (ESS). ${ }^{20-23}$

The association between diabetes and thyroid dysfunction including hyperthyroidism and hypothyroidism was well established in previous literature. 6,7 Our study is in line with another cross-sectional study of type 2 diabetes in China $^{24}$ and an observational study of type 1 diabetes in Poland. $^{25}$ Interestingly, two longitudinal studies from Korea did not prove a statistically significant association between the incidence of diabetes and the suppressed TSH level, but the individual-level changes of $\mathrm{TSH}^{26,27}$ However, a cohort study from Rotterdam suggested that higher TSH levels and lower FT4 levels within the reference range of thyroid function were associated with a higher risk of diabetes, ${ }^{15}$ while another cohort study in the Netherlands showed no association between plasma TSH levels and incident T2DM. ${ }^{28}$ Cross-sectional studies in Korea and Peru suggested increased FT3 and FT3/FT4 ratio are associated with metabolic syndrome and insulin resistance. $^{29-31}$ The difference in these results may be explained by the study population. All participants with thyroid autoimmunity were excluded from our analysis. They are found in over $10 \%$ of the population and likely to develop early-stage thyroid dysfunction, which may elevate or reduce the TSH levels. None of the previous studies excluded the impact of thyroid autoimmunity, 


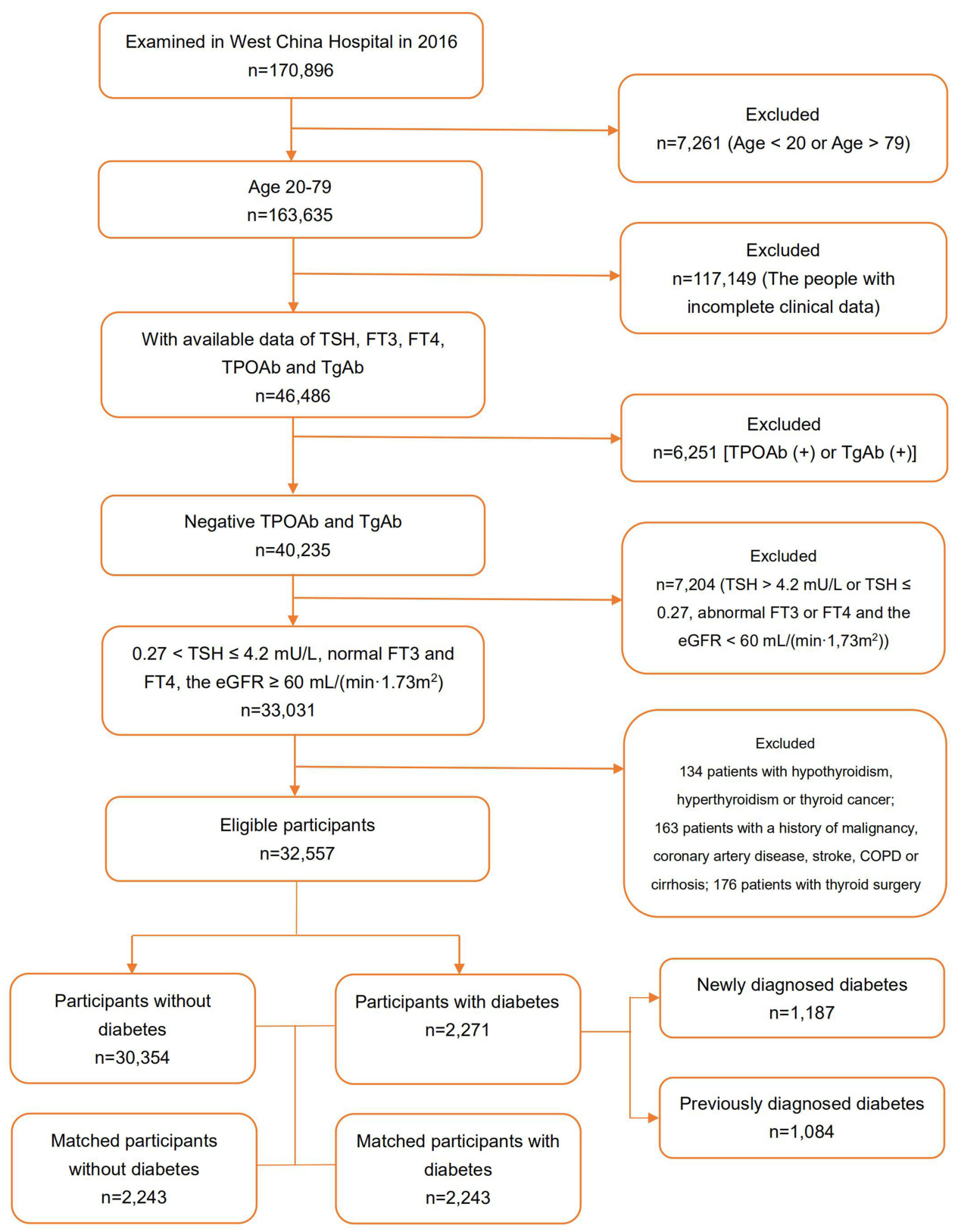

Figure I Screening process of the participants.

Abbreviations: TSH, thyroid stimulating hormone; FT4, free thyroxine; FT3, free triiodothyronine; TPOAb, thyroid peroxidase antibody; TGAb, thyroglobulin antibody; COPD, chronic obstructive pulmonary disease; eGFR, estimated glomerular filtration rate.

which may slightly affect the results. ${ }^{15,26-28}$ To be noted, two cohort studies from the Netherlands included Caucasian adults with higher cardiovascular risks than our study population, ${ }^{15,28}$ while the Korean study was more in line with us. ${ }^{26,27}$ It suggested this association could be ethnicity-based and needed more validation in different populations.

The suppressed TSH level in the PDD group may be partially explained by the increased risk of ESS and metformin therapy in this population. ${ }^{9,32,33}$ Changes in thyroid hormone metabolism due to altered type 1,2 and 3 deiodinase may underlie the observation since these enzymes had shown altered activity in models of disease or inflammation, ${ }^{34,35}$ meaning the FT3 level declined and the FT4/FT3 ratio evaluated as the duration grew, indicating the typical change of $\mathrm{ESS}^{36}$ along with the deterioration of diabetes. It suggests periodic screening of thyroid hormones in euthyroid patients with diabetes may not be 
Table I Baseline Characteristics of Participants

\begin{tabular}{|c|c|c|c|c|c|c|c|}
\hline \multirow[t]{2}{*}{ Parameter } & \multirow{2}{*}{$\begin{array}{l}\text { Control } \\
(n=30,354)\end{array}$} & \multicolumn{3}{|l|}{$D M(n=227 I)$} & \multirow{2}{*}{$\begin{array}{l}\text { Matched Control } \\
(n=2,243)\end{array}$} & \multirow{2}{*}{$\begin{array}{l}\text { Matched DM } \\
(n=2,243)\end{array}$} & \multirow[t]{2}{*}{$\mathbf{P}$} \\
\hline & & $\begin{array}{l}\text { NDD } \\
(n=I, 187)\end{array}$ & $\begin{array}{l}\text { PDD } \\
(n=1,084)\end{array}$ & $\mathbf{P}$ & & & \\
\hline Age (yr, SD) & $43.30 \pm 11.01$ & $51.66 \pm 9.96$ & $55.84 \pm 9.51$ & $<0.001$ & $53.42 \pm 9.94$ & $53.54 \pm 9.85$ & 0.68 \\
\hline Female/case (\%) & $12,212(40.32)$ & $207(17.44)$ & $227(20.94)$ & 0.03 & $422(18.81)$ & $422(\mid 8.8 I)$ & $>0.99$ \\
\hline BMI $\left(\mathrm{kg} / \mathrm{m}^{2}, \mathrm{SD}\right)$ & $23.58 \pm 3.22$ & $26.08 \pm 3.18$ & $24.97 \pm 2.91$ & $<0.001$ & $25.45 \pm 2.98$ & $25.47 \pm 2.98$ & 0.79 \\
\hline$W C(\mathrm{~cm}, \mathrm{SD})$ & $80.21 \pm 10.03$ & $89.17 \pm 8.88$ & $86.96 \pm 8.69$ & $<0.001$ & $86.76 \pm 8.49$ & $87.97 \pm 8.64$ & $<0.001$ \\
\hline Current smoking/case (\%) & 7,356 (24.29) & $474(39.93)$ & $352(32.47)$ & $<0.001$ & $25(1.11)$ & $819(36.5 \mathrm{I})$ & $<0.001$ \\
\hline Current drinking/case (\%) & 3,630 (II.99) & $263(22.16)$ & $175(16.14)$ & $<0.001$ & $12(5.35)$ & 437 (19.48) & $<0.001$ \\
\hline Hypertension/case (\%) & I,89| (6.24) & $239(20.13)$ & $318(29.34)$ & $<0.001$ & $333(14.85)$ & $54 \mid(24.12)$ & $<0.001$ \\
\hline $\mathrm{SBP}(\mathrm{mmHg}, \mathrm{SD})$ & $117.19 \pm 15.54$ & $|29.57 \pm 17.7|$ & $127.88 \pm 17.24$ & 0.02 & $125.10 \pm 15.77$ & $128.58 \pm 17.46$ & $<0.001$ \\
\hline $\mathrm{DBP}(\mathrm{mmHg}, \mathrm{SD})$ & $74.00 \pm 10.61$ & $80.70 \pm 11.51$ & $76.95 \pm 10.46$ & $<0.001$ & $77.96 \pm 10.28$ & $78.88 \pm 11.16$ & 0.004 \\
\hline $\mathrm{FPG}(\mathrm{mmol} / \mathrm{L}, \mathrm{SD})^{\mathrm{a}}$ & $4.99 \pm 0.52$ & $8.30 \pm 2.66$ & $8.68 \pm 3.03$ & 0.01 & $5.15 \pm 0.55$ & $8.49 \pm 2.86$ & $<0.001$ \\
\hline $\mathrm{HbAlc}(\%, \mathrm{SD})^{\mathrm{a}}$ & $5.42 \pm 0.35$ & $7.34 \pm 1.38$ & $7.45 \pm 1.59$ & 0.22 & $5.56 \pm 0.36$ & $7.40 \pm 1.49$ & $<0.001$ \\
\hline $\mathrm{TG}(\mathrm{mmol} / \mathrm{L}, \mathrm{SD})^{\mathrm{a}}$ & $1.51 \pm 1.20$ & $2.74 \pm 2.42$ & $2.01 \pm 1.74$ & $<0.001$ & $1.67 \pm 1.073$ & $2.40 \pm 2.16$ & $<0.001$ \\
\hline LDL-C (mmol/L, SD) & $2.74 \pm 0.73$ & $2.94 \pm 0.81$ & $2.76 \pm 0.79$ & $<0.001$ & $2.90 \pm 0.72$ & $2.85 \pm 0.81$ & $<0.001$ \\
\hline $\mathrm{HDL}-\mathrm{C}(\mathrm{mmol} / \mathrm{L}, \mathrm{SD})^{\mathrm{a}}$ & $1.41 \pm 0.40$ & $1.19 \pm 0.34$ & $1.26 \pm 0.37$ & $<0.001$ & $1.31 \pm 0.34$ & $1.21 \pm 0.35$ & $<0.001$ \\
\hline $\mathrm{TSH}(\mathrm{mU} / \mathrm{L}, \mathrm{SD})$ & $2.29 \pm 0.87$ & $2.26 \pm 0.89$ & $2.28 \pm 0.88$ & 0.69 & $2.35 \pm 0.89$ & $2.27 \pm 0.88$ & 0.001 \\
\hline FT4 (pmol/L, SD) & $16.90 \pm 2.31$ & $16.96 \pm 2.30$ & $|7.23 \pm 2.4|$ & 0.01 & $16.62 \pm 2.22$ & $17.09 \pm 2.35$ & 0.001 \\
\hline FT3 (pmol/L, SD) ${ }^{a}$ & $5.06 \pm 0.86$ & $5.11 \pm 1.02$ & $4.88 \pm 0.88$ & $<0.001$ & $5.07 \pm 0.65$ & $5.00 \pm 0.97$ & $<0.001$ \\
\hline FT4/FT3 (SD) & $3.38 \pm 0.48$ & $3.38 \pm 0.56$ & $3.58 \pm 0.55$ & $<0.001$ & $3.31 \pm 0.48$ & $3.47 \pm 0.56$ & 0.004 \\
\hline $\operatorname{ALT}(I U / L, S D)^{a}$ & $27.98 \pm 24.99$ & $38.24 \pm 32.53$ & $29.77 \pm 18.33$ & $<0.001$ & $31.35 \pm 23.80$ & $34.11 \pm 27.06$ & $<0.001$ \\
\hline AST (IU/L, SD) ${ }^{\mathrm{a}}$ & $25.96 \pm 15.00$ & $30.23 \pm 17.58$ & $26.39 \pm 13.37$ & $<0.001$ & $27.85 \pm|2.9|$ & $28.32 \pm 15.83$ & $<0.14$ \\
\hline GGT (IU/L, SD) $)^{\mathrm{a}}$ & $33.85 \pm 42.95$ & $69.38 \pm 119.11$ & $43.92 \pm 94.03$ & $<0.001$ & $36.33 \pm 40.82$ & $57.32 \pm 109.16$ & $<0.001$ \\
\hline $\begin{array}{l}\text { eGFR }\left(\mathrm{mL} /\left(\min \cdot 1.73 \mathrm{~m}^{2}\right)\right. \\
\mathrm{SD})^{\mathrm{a}}\end{array}$ & $97.03 \pm 17.55$ & $89.99 \pm 14.33$ & $89.13 \pm 13.58$ & 0.22 & $86.33 \pm 14.25$ & $89.64 \pm 13.98$ & $<0.001$ \\
\hline
\end{tabular}

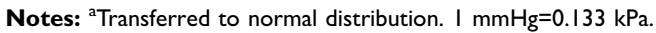

Abbreviations: TSH, thyroid stimulating hormone; FT4, free thyroxine; FT3, free triiodothyronine; TPOAb, thyroid peroxidase antibody; TGAb, thyroglobulin antibody; $\mathrm{HbAlc}$, hemoglobin Alc; COPD, chronic obstructive pulmonary disease; eGFR, estimated glomerular filtration rate; BMI, body mass index; FPG, fasting plasma glucose; TG, triglyceride; LDL-C, low-density lipoprotein cholesterol; HDL-C, high-density lipoprotein cholesterol; ALT, alanine aminotransferase; AST, aspartate aminotransferase; GGT, glutamyl transpeptidase; DBP, diastolic blood pressure; SBP, systolic blood pressure; WC, waist circumstance; DM, diabetes mellitus; NDD, newly diagnosed as diabetes; PDD, previously diagnosed as diabetes.

Table 2 The Association Between Diabetes and Thyroid Parameters

\begin{tabular}{|c|c|c|c|c|c|c|}
\hline & \multicolumn{2}{|l|}{ Model I } & \multicolumn{2}{|l|}{ Model 2} & \multicolumn{2}{|l|}{ Model 3} \\
\hline & OR $(95 \% \mathrm{CI})$ & $P$ & OR $(95 \% \mathrm{Cl})$ & $P$ & OR $(95 \% \mathrm{CI})$ & $P$ \\
\hline TSH & $0.90(0.84-0.96)$ & 0.001 & $0.88(0.82-0.94)$ & $<0.001$ & $0.88(0.82-0.95)$ & $<0.001$ \\
\hline FT4 & $1.10(1.07-1.13)$ & $<0.001$ & I.II (I.08-I.14) & $<0.001$ & I.II (I.08-I.I4) & $<0.001$ \\
\hline FT3 & $0.89(0.82-0.97)$ & $<0.001$ & $0.86(0.78-0.93)$ & $<0.001$ & $0.85(0.78-0.93)$ & $<0.001$ \\
\hline FT4/FT3 & $1.84(1.64-2.07)$ & $<0.001$ & $2.02(1.79-2.28)$ & $<0.001$ & $2.05(1.8 I-2.32)$ & $<0.001$ \\
\hline
\end{tabular}

Notes: Model I, univariate; Model 2, DBP and TG; Model 3, DBP, TG, ALT and eGFR.

Abbreviations: TSH, thyroid stimulating hormone; FT4, free thyroxine; FT3, free triiodothyronine; DBP, diastolic blood pressure; eGFR, estimated glomerular filtration rate; TG, triglyceride; ALT, alanine aminotransferase.

necessary since ESS is an adaptive change of peripheral turnover of thyroid hormones.

Our study has some strengths. Firstly, the study enrolled a large population of confirmed euthyroid adults, which may better reflect the pathophysiological changes of thyroid hormones in patients with diabetes. Secondly, controls were selected by 1:1 matching and three multivariate models in the study showed consistent results, which suggested the robustness of our results.

However, our study has some limitations. Firstly, this is a cross-sectional study, which cannot conclude the causation between thyroid hormone profiles and diabetic development. Secondly, the study population was recruited from the health examination participants, possibly different 
Table 3 The Difference in Thyroid Parameters Between Newly Diagnosed Diabetes and Previously Diagnosed Diabetes

\begin{tabular}{|c|c|c|c|c|c|c|c|c|}
\hline & \multicolumn{2}{|l|}{ Model I } & \multicolumn{2}{|l|}{ Model 2} & \multicolumn{2}{|l|}{ Model 3} & \multicolumn{2}{|l|}{ Model 4} \\
\hline & OR (95\% CI) & $P$ & OR (95\% CI) & $P$ & OR (95\% CI) & $P$ & OR (95\% CI) & $P$ \\
\hline TSH & $1.02(0.93-I .12)$ & 0.69 & $1.00(0.9 I-I .10)$ & 0.98 & I.0I (0.9I-I.II) & 0.91 & 1.01 (0.92-I.I2) & 0.78 \\
\hline FT4 & $1.05(1.01-1.09)$ & 0.01 & $1.08(1.04-1.12)$ & $<0.001$ & $1.08(1.04-1.12)$ & $<0.001$ & $1.08(1.04-1.12)$ & $<0.001$ \\
\hline FT3 & $0.67(0.59-0.76)$ & $<0.001$ & $0.80(0.70-0.91)$ & 0.001 & $0.79(0.69-0.90)$ & $<0.001$ & $0.81(0.71-0.92)$ & 0.002 \\
\hline FT4/FT3 & $1.97(1.68-2.31)$ & $<0.001$ & $1.80(1.52-2.12)$ & $<0.001$ & $1.82(1.54-2.15)$ & $<0.001$ & $1.76(1.49-2.08)$ & $<0.001$ \\
\hline
\end{tabular}

Notes: Model I, none (univariate); Model 2, age, sex, BMI; Model 3, age, sex, BMI, DBP and TG; Model 4, age, sex, BMI, DBP, TG, ALT and eGFR.

Abbreviations: TSH, thyroid stimulating hormone; FT4, free thyroxine; FT3, free triiodothyronine; DBP, diastolic blood pressure; BMI, body mass index; eGFR, estimated glomerular filtration rate; TG, triglyceride; ALT, alanine aminotransferase.

from the community-based population. Thirdly, we could not access the detailed duration of diabetes or the undergoing treatment of the patients, which might be unadjusted biases in the study, but we could conclude the duration of PDD was longer than that of NDD, since all patients in NDD were diagnosed in 2016, while all patients in PDD were diagnosed before 2016. Fourthly, we did not differentiate type 1 and type 2 diabetes because of the limitation of the patient information. Nonetheless, type 2 diabetes was likely to be demonstrative according to the epidemiology of diabetes in China. Finally, we did not evaluate the levels of rT3, which may help confirm the conversive changes of the thyroid hormones but is unavailable in the data source.

\section{Conclusion}

Our study found an elevated FT4/FT3 ratio in euthyroid patients with newly or previously diagnosed diabetes, especially in the latter, suggesting alterations in thyroid hormone turnover in these patients.

\section{Abbreviations}

NDD, newly diagnosed with diabetes; PDD, previously diagnosed with diabetes; TSH, thyroid stimulating hormone; FT4, free thyroxine; FT3, free triiodothyronine; ORs, odds ratios; CI, confidence interval; TPOAb, thyroid peroxidase antibody; TGAb, thyroglobulin antibody; HbA1c, hemoglobin A1c; COPD, chronic obstructive pulmonary disease; eGFR, estimated glomerular filtration rate; BMI, body mass index; FPG, fasting plasma glucose; TG, triglyceride; LDL-C, low-density lipoprotein cholesterol; HDL-C, high-density lipoprotein cholesterol; ALT, alanine aminotransferase; AST, aspartate aminotransferase; GGT, glutamyl transpeptidase; DBP, diastolic blood pressure; SBP, systolic blood pressure; WC, waist circumstance; DM, diabetes mellitus; rT3, reverse triiodothyronine; D1, type 1 deiodinases; ESS, euthyroid sickness syndrome; T4, tetraiodothyronine; T3, triiodothyronine.

\section{Data Sharing Statement}

The datasets used and/or analyzed during the current study are available from the corresponding author, Sheyu $\mathrm{Li}$, on reasonable request.

\section{Ethics Approval and Consent to Participate}

This study is approved by the ethical committee of West China Hospital, Sichuan University (No. 2015-202). Subjects were only included if they signed informed consent agreeing to the scientific use of their health data.

\section{Author Contributions}

All authors made substantial contributions to conception and design, acquisition of data, or analysis and interpretation of data; took part in drafting the article or revising it critically for important intellectual content; gave final approval of the version to be published; and agree to be accountable for all aspects of the work.

\section{Funding}

This study was supported by 1.3.5 Project for Disciplines of Excellence, West China Hospital, Sichuan University (grant number ZYGD18022) and Sichuan Science and Technology Program (grant numbers 2017RZ0046, 2018SZ0087 and 2019YFH0150). Sheyu Li has also received grants from the National Natural Science Foundation of China (grant number 81400811 and 21534008).

\section{Disclosure}

The authors have no conflicts of interest to declare. 


\section{References}

1. McAninch E, Bianco A. Thyroid hormone signaling in energy homeostasis and energy metabolism. Ann N Y Acad Sci. 2014;1311:77-87. doi:10.1111/nyas. 12374

2. Meruvu S, Ayers SD, Winnier G, Webb P. Thyroid hormone analogues: where do we stand in 2013? Thyroid. 2013;23(11):1333-1344. doi:10.1089/thy.2012.0458

3. Sinha RA, Singh BK, Yen PM. Thyroid hormone regulation of hepatic lipid and carbohydrate metabolism. Trends Endocrinol Metab. 2014;25(10):538-545. doi:10.1016/j.tem.2014.07.001

4. Alkemade A. Central and peripheral effects of thyroid hormone signalling in the control of energy metabolism. J Neuroendocrinol. 2010;22(1):56-63. doi:10.1111/j.1365-2826.2009.01932.x

5. Warner A, Mittag J. Thyroid hormone and the central control of homeostasis. J Mol Endocrinol. 2012;49(1):R29-R35. doi:10.1530/ JME-12-0068

6. Díez J, Iglesias P. Subclinical hyperthyroidism in patients with type 2 diabetes. Endocrine. 2012;42(1):157-163. doi:10.1007/s12020-0129621-3

7. Han C, He X, Xia X, et al. Subclinical hypothyroidism and type 2 diabetes: a systematic review and meta-analysis. PLoS One. 2015;10 (8):e0135233. doi:10.1371/journal.pone.0135233

8. Distiller L, Polakow E, Joffe B. Type 2 diabetes mellitus and hypothyroidism: the possible influence of metformin therapy. Diabet Med. 2014;31(2):172-175. doi:10.1111/dme.12342

9. Furukawa S, Yamamoto S, Todo Y, et al. Association between subclinical hypothyroidism and diabetic nephropathy in patients with type 2 diabetes mellitus. Endocr J. 2014;61(10):1011-1018. doi:10.1507/endocrj.EJ14-0206

10. Khatiwada S, KC R, Sah S, et al. Thyroid dysfunction and associated risk factors among nepalese diabetes mellitus patients. Int $J$ Endocrinol. 2015;2015:1-5. doi:10.1155/2015/570198

11. Ashwini S, Bobby Z, Joseph M. Mild hypothyroidism improves glucose tolerance in experimental type 2 diabetes. Chem Biol Interact. 2015;235:47-55. doi:10.1016/j.cbi.2015.04.007

12. Misiti S, Anastasi E, Sciacchitano S, et al. 3,5,3'-Triiodo-L-thyronine enhances the differentiation of a human pancreatic duct cell line (hPANC-1) towards a beta-cell-Like phenotype. J Cell Physiol. 2005;204(1):286-296. doi:10.1002/jep.20293

13. Furuya F, Shimura H, Yamashita S, Endo T, Kobayashi T. Liganded thyroid hormone receptor-alpha enhances proliferation of pancreatic beta-cells. J Biol Chem. 2010;285(32):24477-24486. doi:10.1074/ jbc.M109.100222

14. Falzacappa C, Panacchia L, Bucci B, et al. 3,5,3'-triiodothyronine (T3) is a survival factor for pancreatic beta-cells undergoing apoptosis. J Cell Physiol. 2006;206(2):309-321. doi:10.1002/ jcp. 20460

15. Chaker L, Ligthart S, Korevaar T, et al. Thyroid function and risk of type 2 diabetes: a population-based prospective cohort study. $B M C$ Med. 2016;14(1):150. doi:10.1186/s12916-016-0693-4

16. American Diabetes Association. 2. classification and diagnosis of diabetes: standards of medical care in diabetes-2018. Diabetes Care. 2018;41(Supplement 1):S13-S27. doi:10.2337/ dc18-S002

17. Levey A, Stevens L, Schmid C, et al. A new equation to estimate glomerular filtration rate. Ann Intern Med. 2009;150(9):604-612. doi:10.7326/0003-4819-150-9-200905050-00006

18. Tarcin O, Abanonu GB, Yazici D, Tarcin O. Association of metabolic syndrome parameters with TT3 and FT3/FT4 ratio in obese Turkish population. Metab Syndr Relat Disord. 2012;10(2):137-142. doi:10.1089/met.2011.0098

19. Hulbert A. Thyroid hormones and their effects: a new perspective. Biol Rev Camb Philos Soc. 2000;75(4):519-631. doi:10.1017/ S146479310000556X
20. Lee S, Farwell A. Euthyroid sick syndrome. Compr Physiol. 2016;6 (2):1071-1080.

21. Umpierrez G. Euthyroid sick syndrome. South Med J. 2002;95 (5):506-513. doi:10.1097/00007611-200295050-00007

22. Moura Neto A, Zantut-Wittmann D. Abnormalities of thyroid hormone metabolism during systemic illness: the low $\mathrm{t} 3$ syndrome in different clinical settings. Int $J$ Endocrinol. 2016;2016:2157583. doi: $10.1155 / 2016 / 2157583$

23. Moura Neto A, Parisi MC, Alegre SM, Pavin EJ, Tambascia MA, Zantut-Wittmann DE. Relation of thyroid hormone abnormalities with subclinical inflammatory activity in patients with type 1 and type 2 diabetes mellitus. Endocrine. 2016;51(1):63-71. doi:10.1007/ s12020-015-0651-5

24. Gu Y, Li H, Bao X, et al. The relationship between thyroid function and the prevalence of type 2 diabetes mellitus in euthyroid subjects. J Clin Endocrinol Metab. 2017;102(2):434-442. doi:10.1210/jc.2016-2965

25. Falkowski B, Rogowicz-Frontczak A, Grzelka A, et al. Higher free triiodothyronine concentration is associated with lower prevalence of microangiopathic complications and better metabolic control in adult euthyroid people with type 1 diabetes. Endocrine. 2018;60(3):458465. doi:10.1007/s12020-018-1582-8

26. Jun J, Jin S, Jee J, et al. TSH increment and the risk of incident type 2 diabetes mellitus in euthyroid subjects. Endocrine. 2017;55(3):944953. doi:10.1007/s12020-016-1221-1

27. Jun J, Jee J, Bae J, et al. Association between changes in thyroid hormones and incident type 2 diabetes: a seven-year longitudinal study. Thyroid. 2017;27(1):29-38. doi:10.1089/thy.2016.0171

28. de Vries T, Kappelle L, van der Graaf Y, et al. Thyroid-stimulating hormone levels in the normal range and incident type 2 diabetes mellitus. Acta Diabetol. 2019;56(4):431-440. doi:10.1007/s00592018-1231-y

29. Dimic D, Golubovic MV, Radenkovic S, Radojkovic D, Pesic M. The effect of metformin on TSH levels in euthyroid and hypothyroid newly diagnosed diabetes mellitus type 2 patients. Bratisl Lek Listy. 2016;117(8):433-435. doi:10.4149/bll_2016_084

30. Park SY, Park SE, Jung SW, et al. Free triiodothyronine/free thyroxine ratio rather than thyrotropin is more associated with metabolic parameters in healthy euthyroid adult subjects. Clin Endocrinol (Oxf). 2017;87(1):87-96. doi:10.1111/cen.13345

31. Urrunaga-Pastor D, Guarnizo-Poma M, Moncada-Mapelli E, et al. High free triiodothyronine and free-triiodothyronine-to-free-thyroxine ratio levels are associated with metabolic syndrome in a euthyroid population. Diabetes Metab Syndr. 2018;12(2):155-161. doi:10.1016/ j.dsx.2017.12.003

32. Benites-Zapata VA, Urrunaga-Pastor D, Torres-Mallma C, PradoBravo C, Guarnizo-Poma M, Lázaro-Alcántara H. Is free triiodothyronine important in the development of insulin resistance in healthy people? Diabetes Metab Syndr. 2017;11(Suppl 2):S663-S667.

33. Lupoli R, Di Minno A, Tortora A, Ambrosino P, Lupoli GA, Di Minno MN. Effects of treatment with metformin on TSH levels: a meta-analysis of literature studies. J Clin Endocrinol Metab. 2014;99 (1):E143-E148. doi:10.1210/jc.2013-2965

34. Wajner SM, Maia AL. New insights toward the acute non-thyroidal illness syndrome. Front Endocrinol (Lausanne). 2012;3:8. doi:10.3389/fendo.2012.00008

35. Jakobs TC, Mentrup B, Schmutzler C, Dreher I, Korle J. Proinflammatory cytokines inhibit the expression and function of human type I 5'-deiodinase in HepG2 hepatocarcinoma cells. Eur $J$ Endocrinol. 2002;146(4):559-566. doi:10.1530/eje.0.1460559

36. Köhrle J. Thyroid hormone transporters in health and disease: advances in thyroid hormone deiodination. Best Pract Res Clin Endocrinol Metab. 2007;21(2):173-191. doi:10.1016/j.beem.2007.04.001 


\section{Publish your work in this journal}

Diabetes, Metabolic Syndrome and Obesity: Targets and Therapy is an international, peer-reviewed open-access journal committed to the rapid publication of the latest laboratory and clinical findings in the fields of diabetes, metabolic syndrome and obesity research. Original research, review, case reports, hypothesis formation, expert opinion and commentaries are all considered for publication. The manuscript management system is completely online and includes a very quick and fair peer-review system, which is all easy to use. Visit http://www.dovepress.com/testimonials.php to read real quotes from published authors.

Submit your manuscript here: https://www.dovepress.com/diabetes-metabolic-syndrome-and-obesity-targets-and-therapy-journal 